\section{Molecular Markers in Peripheral Blood of Patient with Acute Myocardial Infarction}

Marcelo Rodrigues Bacci' ${ }^{1}$, Mariana Jancis Rigolo ${ }^{1}$, Flavia Gehrke', Neif Murad², Livia Yadoya Vasconcelos ${ }^{1}$, Fernando Luiz Affonso Fonseca ${ }^{1}$

\title{
Abstract
}

Alms: Cyclooxygenases (COX) are involved in inflammation and in prostaglandin metabolism. It is estimated that the use of blockers causes a steep rise in cardiovascular events when compared with patients who did not use them. The aim of this report is to show the association of the increase in COX-2 expression in relation to time in a patient with acute myocardial infarction (AMI).

Methods: A 54-year-old black woman, hypertensive and ex-cocaine user, presented with typical precordial pain and ischemia in the anterior wall of the myocardium identified by an ECG. Serial blood collections were performed in order to evaluate COX-2 and NF-kB gene expressions. Forty days prior the event she had been submitted to percutaneous coronary angioplasty due to AMI. The coronarlography revealed stent thrombosis.

Results: COX-2 and NF-kB expressions peaked in the first 24 hours after the thrombotic event.

Discussion: There is an increase in COX-2 expression on atherosclerotic plaques. The use of COX-2 blockers increase the risk of ischemia given their vasoconstrictor effect, resulting in a decrease in oxygen supply to the myocardium. In sum, the increase in COX-2 expression was associated with acute coronary event in this patient.

\section{Introduction}

Cyclooxygenases are enzymes directly involved in the inflammatory process. Once stimulated, the arachidonic acid is turned into prostaglandins by the cyclooxygenases. Prostaglandins, in turn, take part

1 General Practice Department of $A B C$ Medical School.

2 Cardiology Department of ABC Medical School.

Contact information:

Marcelo Rodrigues Bacci.

Address: Av. Principe de Gales, 821. Santo Andre, Brazil. ZIP 09060-650.

Tel: +55 11981937005 .

झ" mrbacci@yahoo.com

Keywords

Cyclooxygenase; Acute

Myocardial Infarction;

Atherosclerosis. 
in platelet activation, vasoconstriction, vascular endothelial regulation, gastrointestinal mucosal protection, brochodilation, kidney function and inflammatory processes. Recent studies have revealed an important association of COX-2 with cardiovascular protection. Shinmura demonstrated in unanesthetized mice that COX-2 acts in the regulation of these effects during a late stage of myocardial preconditioning. [1]

COX-2 blockers are presumed to cause a steep rise in cardiovascular events, like acute myocardial infarction and angina, when compared with patients who did not use them2. This effect suggests a protective effect of COX-2 in individuals who do not have it artificially inhibited, like when in use of NSAID [2].

NF-kB acts as a central signaling pathway of several other inflammatory pathways [3]. Thus, it jointly acts with the release of COX and its isoforms.

Myocyte cell death in acute myocardial infarction events may release fragments of DNA (DNA-free fraction in the plasma), and this fact may be related to a higher NF-kB expression with a consequent strengthening of this inflammatory pathway.

The aim of this report is to show the association of the increase in COX-2 expression in relation to time during the evolution of patient with acute coronary syndrome.

\section{Case Summary}

A female fifty-three year old patient, smoker and hypertensive, regularly using enalapril and hydrochlorothiazide in optimal doses. An occasional cocaine user, the patient was admitted with typical precordial pain diagnosed as non-ST-segment elevation acute myocardial infarction. The case was analyzed by means of a coronary angiography, and a critical lesion in the medial segment of the left anterior descending artery was identified. Conventional stent angioplasty was performed and the patient was dismissed seven days later.
Forty-five days after first hospital admission, the patient returned with similar clinical features; however, this time the pain was more severe in the precordial region, and the diagnosis was ST-segment elevation anterior wall AMI, Killip class I. With the patient's consent, after the performance of a new contrast-enhanced coronary angiography, blood samples were collected at time points $0,6,12$ and 24 hours on the second day after the AMI event. After blood collection, RNA was extracted and cDNA synthesis was performed. Next, cDNA was amplified to COX-2 and NF-kB gene expressions using their specific primers. The angiography revealed previously implanted stent thrombosis. The patient stated not having started the prescribed double antiaggregant therapy.

Figure 1: Analysis of COX-2 and NF-kB gene expressions in plasma DNA concentration in patient with acute coronary syndrome after the use of venous iodinated contrast.

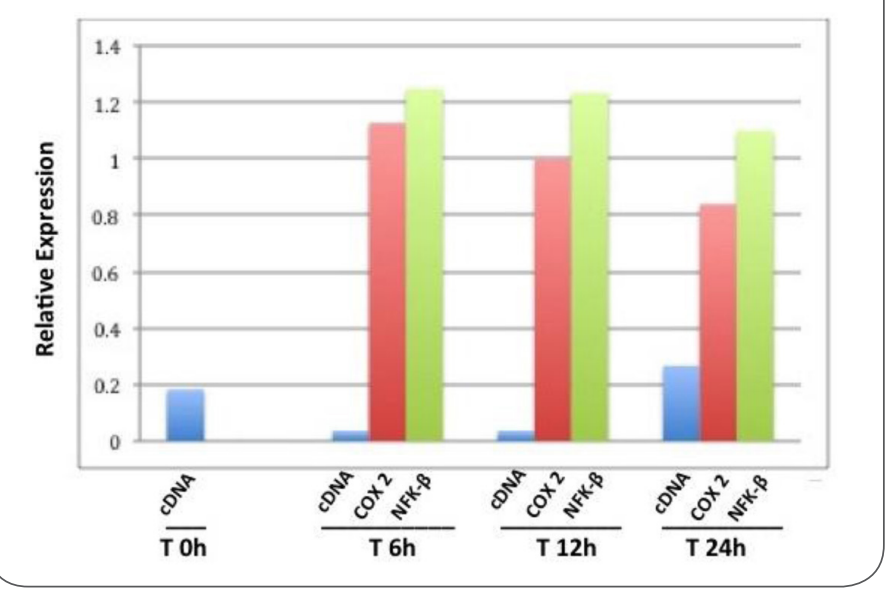

\section{Discussion}

Besides all the studies on the use of NSAIDs, others involving COX-2 polymorphisms, like the one conducted by Vogel [3], also suggest an important relation between COX-2 and acute coronary syndrome (ACS). The study showed that COX-2 polymorphism represents a higher risk of ACS, especially in males, which confirms this relation. 
The current case report may be analyzed according to different perspectives: the difficulties patients face in adhering to the treatment due to socio-economic reasons that impact on specific medication acquisition; the event of post-angioplasty thrombosis and its differentiation from in-stent stenosis; the role of the contrast as an endothelial aggression factor due to its osmolarity and viscosity. COX-2 plays an important role in myocardial protection once it maintains the vasodilation necessary for the maintenance of myocardial oxygen supply $[4,5]$. Its higher expression, as shown in this report, confirms the protective mechanism described above [5].

On the other hand, increased NF-kB expression confirms the inflammatory mechanism in AMI events. NF-kB plays different roles that regulate from pro-inflammatory to protective factors like the stimulus for nitric oxide synthase. [4]

A different behavior of the genes could be observed when the patient was diagnosed with nonST-segment elevation AMI on her first admission, during which she underwent a contrast-enhanced angiography. Therefore, it may be inferred that the ST-segment elevation AMI event was the determinant factor for the peak detection of COX-2 and NF-kB gene expressions in patient undergoing STsegment elevation AMI with stent thrombosis due to poor medication adherence after the performance of a coronary angiography.

In conclusion, COX-2 expression correlates with higher activity during AMI evolution as a protective response against ischemia.

\section{Conflict of Interests}

No conflict of interests to report.

\section{References}

1. Shinmura K, Tang XL, Wang Y, Xuan YT, LIU SQ, Takano H, et al. Cyclooxygenase-2 mediates the cardioprotective effects of the late phase os ischemic preconditioning conscious rabbits. Proc Natl Acad Sci USA. 2000; 97: 10197-202

2. Bresalier RS, Sandler RS, Quan H, Bolognese JA, Laine L. MEDAL Steering Committee. Clinical trial desing and pacientes demographics of the Multinational Etoricoxib and Diclofenac Arthritis Long-Term (MEDAL) study progam: cardiovascular outcomes with etoricoxib versus diclofenac in patientes with osteoarthritis and rheumatoid arthritis. Am Heart J. 2006; 152:237-45.

3. Vogel U, Segel S, Dethlefsen C, Tjonneland A, Saber AT, Wallin $\mathrm{H}$, et al. Associations between COX-2 polymorphisms, blood cholesterol and risk of acute coronary syndrome. Artherosclerosis 209. 2020; 155:162.

4. Frangogiannis NG, Smith CW, Entman ML. The inflammatory response in myocardial infarction. Cardiovasc Res. 2002 Jan;53(1):31-47.

5. Fang L, Moore XL, Dart AM, Wang LM. Systemic inflammatory response following acute myocardial infarction. J Geriatr Cardiol. 2015 May;12(3):305-12. doi: 10.11909/j.issn.16715411.2015.03.020.

\section{Publish in International Archives of Medicine}

International Archives of Medicine is an open access journal publishing articles encompassing all aspects of medical science and clinical practice. IAM is considered a megajournal with independent sections on all areas of medicine. IAM is a really international journal with authors and board members from all around the world. The journal is widely indexed and classified Q1 in category Medicine. 